\title{
CRISIS DE LA SEGUNDA REPÚBLICA ESPAÑOLA (UN ANÁLISIS OBJETIVO 75 AÑOS DESPUÉS)
}

\author{
POR \\ MANUEL RAMÍREZ JIMÉNEZ \\ Catedrático de Derecho Político \\ Universidad de Zaragoza
}

\section{INTRODUCCIÓN}

Durante el año en que estas líneas se escriben, nuestro país, por iniciativa de instancias oficiales está celebrando el 75 aniversario de la proclamación de la Segunda República Española. Se han venido sucediendo gran cantidad de eventos (conferencias, congresos, artículos, programas televisivos, etc) poniendo de manifiesto lo que supuso aquel régimen político y acercando lo que fue sobre todo a generaciones que no lo vivieron.

Escaso reparo hay que hacer a esta iniciativa. La experiencia republicana no podía ni puede quedar en el olvido, ni mucho menos pasar a la historia viciada por las numerosas condenas que el régimen instaurado al finalizar la guerra civil había vertido sobre ella. Durante decenios, fue la gran desconocida o la simplemente tachada de años que habían sumado todos los males posibles. Desde que se pudo, los jóvenes historiadores han venido intentando dar a la luz pública lo que durante decenios había permanecido en la ingrata oscuridad. De este empeño, únicamente puede uno sentir una sincera y merecida satisfacción. 
Pero cuando dicho propósito aspira a poseer carácter científico, lo que, de entrada, se impone es también la objetividad. Ardua tarea contenida en palabras del insigne Marañón: por muy claros y comprobados que queden los hechos, siempre quedará también el tortuoso camino de las interpretaciones. $Y$ es que la objetividad es especialmente difícil cuando se trata de un acontecimiento histórico que terminó en un penoso mar de sangre obra de una cruel guerra civil. Cuando el régimen autoritario subsiguiente se basó, precisamente, en la victoria obtenida en dicha guerra. Cuando todavía pueden coexistir en nuestra hermosa piel de toro tanto quienes durante decenios prestaron sincera lealtad al Ilamado "Nuevo Estado", como quienes conservaban jirones en sus almas por haber servido con mayor o menos entusiasmo a la ilusión republicana. Sí. ¡Ardua tarea la de ser plenamente objetivos!

Pero, a pesar de todo ello, si lo que se pretende es el mayor acercamiento posible a la verdad estudiada y documentada, se impone la necesidad de alejarse tanto de la acomodaticia repulsa cuanto de la alegre «magnificación». Todos los acontecimientos históricos suelen tener zonas de luz y zonas de sombra. Y ambas vertientes tuvo la Segunda República.

El 14 de abril de 1931 advino a España plenamente cubierto de lo primero. De luz. De entusiasmo. Con la República se pretendía poner fin a los muchos años de caciquismo y manipulación del sufragio sobre los que estuvo montada, en gran parte, "el fantasma de la Restauración canovista" como la calificara el maestro Ortega. El imperio de los caciques. La división entre una España oficial y otra España real. La postergación política de la clase obrera y de la nueva izquierda (intelectuales, sobre todo). La Monarquía, encarnada en la persona de Alfonso XIII, había quedado totalmente desprestigiada, incluso entre muchos monárquicos que no hicieron nada para "su salvación", fundamentalmente por dos razones: el haber aceptado la Dictadura de Primo de Rivera y, sobre todo, la constante práctica del llamado «borboneo", es decir su continua intromisión en la vida política amenazando con el recurso a los militares si no se obraba según sus deseos. El famoso "Delenda" que Ortega lanzara en uno de sus artículos constituyó muy posiblemente el aviso final a un Rey que, perdidas unas elecciones municipales y claramente influido en su decisión por el mismo Romanones, opta finalmente por salir de España y dejar el campo libre a la instauración de la República. El país, por lo demás, estaba ya experimentando la influencia de la labor de los intelectuales, unidos en la Agrupación al Servicio de la República, y los principales líderes de los partidos también habían sembrado el camino a través 
de la firma del famoso Pacto de San Sebastián. Todo apuntaba a la llegada de una República que se concebía como "salvadora" de todos los males.

$Y$ en la vertiente o zona positiva tenemos necesariamente que anotar, ante todo, el innegable entusiasmo popular por la llegada y por lo que iba a significar aquel 14 de abril. Llegaba sin pagar a la historia el peaje político de sangre que todo cambio profundo parece obligado a tributar. Sin golpe o pronunciamiento militar que lo partearan. Como consecuencia de unas simples elecciones y la salida del Rey. Con la casi absoluta actitud pasiva de un Ejército tan acostumbrado a inmiscuirse en los asuntos políticos internos. Eran los propios ciudadanos quienes se sentían protagonistas del evento y aplaudían con fuerza al Gobierno Provisional, integrado en parte por personas que salían de la cárcel por haber participado en el Pacto de San Sebastián y que de la cárcel pasaban a la toma del poder. Un 14 de abril que posiblemente no se borre nunca de la historia de nuestro país, incluidos los largos años del posterior exilio. Ya no era el liberalismo avanzado de la Revolución septembrina de 1868. Era mucho más. Y quizá en este "mucho más» es donde, quizá sin percatarse de ello, comenzarían pronto los problemas. Cuando se está discutiendo la Constitución de 1931, estas palabras de nada menos que Jiménez de Asúa, comienzan a sembrar las dudas: "Si la República no hubiera venido a mudarlo todo, no merecería la pena haberla traído" ¡Mudarlo todo! Como si al cambiar un régimen resulte necesario mudar todo lo anterior. Y, claro está, como si eso fuera posible a través de las leyes, por importantes que fueren.

De igual forma, nadie podrá negar la gran labor que en el terreno cultural realizó la Segunda República. En la educación y en la cultura. La obra dejó sus frutos. Durante el primer bienio se anuncia la creación de dieciséis mil escuelas. Hacían falta en un país tan atrasado. Tanto más cuando se había suprimido la función de la enseñanza a las asociaciones religiosas. Se crea la Universidad Internacional Menéndez Pelayo y la Junta para la ampliación de Estudios. Nuestros intelectuales comienzan a cruzar los Pirineos buscando nuevos saberes. La Residencia de Estudiantes se convierte en lugar de convivencia y foro de encuentro de nuestras mejores cabezas. La relación de las mismas será extensa. La influencia krausista se conecta con la reacción intelectual. Blas Carrera, José Giral, José Gaos, Pedro Bosch, José Puche, Mariano Ruiz Funes, Alejandro Otero, Luis Zulueta, José Cuatrecasas, Jiménez de Asúa, Emilio González López, Américo Castro, Sánchez Albornoz, Severo Ochoa, etc, etc. Muchos de ellos hubieron de morir luego en el extranjero. Otros poblaron y dieron riquezas en lugares de Hispanoamé- 
rica. ¡Fantástica e innegable nómina! Europa dejó de ser lo desconocido. En Europa estaba la ciencia y a empaparse de ella fueron muchos de nuestros intelectuales. La advertencia ganivetiana, imitando al Santo, de "No vayas fuera, en el interior de España está la verdad" comenzaba a pasar a la historia.

Pero acaso el problema es que poco de eso llegaba al pueblo todavía inculto. Para éste se fletaron "cositas" que me atrevo a juzgar de escaso resultado. ¿Era suficiente con la Barraca de Federico García Lorca y sus obritas teatrales que juzgo de anodinas y hasta decimonónicas? No. Los verdaderos nombres de la época fueron Valle Inclán, Juan Ramón, Salinas y, sobre todo, Miguel Hernández. Y de esto hubo poco. $Y$ es que, como apuntara Murillo Ferrol hace años, a la República le faltó modernidad. Y la modernidad no podía ni puede quedarse en el "Verde que te quiero verde", algo mucho más cercano a un esteticismo trasnochado que a una España transida de problemas, de muy ricos y muy pobres, de señoritos y de obreros. Esto empañó algo el veredicto. Aunque no se puede poner en duda ni la preocupación por la educación y la cultura, ni su buen resultado frente a una España que había estado mucho tiempo agonizando o bostezando, según el verso de otro gran nombre que también tuvo que pasar los Pirineos poco después.

Y Azaña. Puede que para algún lector cause cierta sorpresa el hecho de incluir a Manuel Azaña en la partida del "haber», de la cara, en estos párrafos sobre la Segunda República. Soy plenamente consciente de que para la derecha de entonces y de buen tracto de la posterior, Azaña recibió el calificativo de "El Monstruo" (por cierto y sin que venga a cuento, idéntico atributo, pero ahora con matices laudatorios, también le colgaron a Manolete, a quien ahora todo el mundillo taurino considera como el mejor torero desde la inmediata postguerra hasta la actualidad y perdón por el paréntesis pero la afición trae estas afirmaciones y otras muchas cuando se habla de política y de toros). Tras haberme empapado de su Memorias verídicas hechas realidad por las manos de Juan Marichal, de quien, sin embargo, discrepo en sus loas a Negrín, muy posiblemente debidas al común origen canario, después de eso, digo, y de no poca lectura de las muchas obras recientemente aparecidas sobre su figura y su obra, tengo para mí que en el personaje de Azaña concurren tres aspectos bastante positivos. Pese a su dudosa consideración como "intelectual», algo desde siempre harto incompatible con el ejercicio de la práctica política, me atrevería a sintetizar esos aspectos de la forma siguiente:

a) Manuel Azaña, por encima de todo, es un gran patriota. Algo que hoy ya no se estila en demasía, pero con lo que muchos coincidimos. 
Vivimos tiempos en los que calificarse de patriota se asimila a ser "facha", no creer mucho en lo de las Autonomías, seguir sintiendo a España como problema y hasta mofarse de eso de que a uno «le siga doliendo España». ¡Qué le vamos a hacer! Desmentir algunas de estas cosas o ratificarse en otras, constituye un menester ajeno al sentido de estas reflexiones. Únicamente diré que en Azaña, como en pocos, estuvo siempre vibrante la advertencia de Ortega: "El español que pretenda huir de las preocupaciones nacionales será hecho prisionero de ellas diez veces al día, y acabará por comprender que para un hombre nacido entre el Bidasoa y Gibraltar es España el problema primario, plenario y perentorio". Curiosamente las tres pes de Ortega, como, según veremos, hubo otro final también con tres pes del mismísimo Azaña. No. Azaña nunca pudo ni quiso huir de las preocupaciones ni problemas de su querida España. Nunca renunció a pronunciar su nombre. Siempre encontramos en sus discursos la palabra por él tan sentida. $Y$ habló de la "Nación española». De la "Patria eterna». De los valores que ésta había aportado al mundo. Del mensaje que España transmitía. De que su República (es decir, la democracia) había de llegar a todos los pueblos de España. A Manuel Azaña el sentimiento español le empapaba su cabeza y su corazón. Estando en el poder y aún más cuando agoniza casi solitariamente en un pueblecito francés.

b) Manuel Azaña supo ser, igualmente, un político práctico. Y moderno. Quizá el más moderno de la República. Nunca basó su actuación en el recurso al pasado. Siempre habló y advirtió sobre el presente y el futuro. El de su República y el de su España. Por eso quiso un Ejército para el futuro. Por eso salva, como solución para el futuro, el problema de las Autonomías. Sabe abdicar de no poco para poder gobernar con los socialistas durante el primer bienio. Pero también sabe imponer e imponerse cuando llegan las pretensiones excesivas de los extremistas. No le tiembla el pulso con una Ley de Defensa de la República, porque bien sabe que eso hoy tan repetido de que la democracia se salva sola es una gran quimera. Lanza a sus juventudes a beber y divulgar los valores propios de la República-Democracia. Se duele al comprobar y afirmar en sus Memorias lo de "He tratado de gobernar mi país con razones y con votos y me han respondido con calumnias y fusiles". Durante mucho tiempo, para gran parte de los ciudadanos todas las virtudes y esperanzas estuvieron depositadas en su persona y en su palabra, cuando superaba todo record en sus famosos discursos a campo abierto. Sí: "Azaña era la República y la República era Azaña». Nunca se dio entonces una asimilación tan generalizada y tan cierta. Incluso más tarde, cuando barrunta la pérdida final de una espantosa guerra, no se limita a dolerse en la intimidad, sino que el 18 de julio de 
1938, desde el Ayuntamiento de Barcelona lanza un discurso que sería su gran legado histórico para la posteridad: "Y cuando la antorcha pase a otras manos, a otros hombres, a otras generaciones, que se acuerden, si alguna vez sienten que les hierve la sangre iracunda y otra vez el genio español vuelve a enfurecerse con la intolerancia y con el odio y con el apetito de destrucción, que piensen en los muertos y que escuchen su lección: la de esos hombres, que han caído embravecidos en la batalla luchando magnánimamente por un ideal grandioso y que ahora, abrigados en la tierra materna, ya no tienen odio, ya no tienen rencor, y nos envían, con los destellos de su luz, tranquila y remota como la de una estrella, el mensaje de la patria eterna que dice a todos sus hijos: Paz, Piedad y Perdón». El Azaña al que en otras ocasiones había perdido su afán por la estética, aquí sí que acierta en lo bello y profundo de este Discurso. No se habla de enemigos, sino de lucha por ideales grandiosos. No es él quien pide: es el mensaje de la patria eterna. Y lo eterno no es únicamente un orgullo por el pasado: es también un aviso a lo porvenir. Sus palabras servían para entonces y siguen sirviendo para ahora. Frenar el impulso ciego. Sesgar la ira. Sin duda está hablando un político que, muchos años después, bien puede servir en los aconteceres de nuestra hora.

Y c) También Manuel Azaña tuvo la virtud de reconocer errores. Los de la paz y, sobre todo, los que llevaron a la guerra. Habría que reproducir íntegramente las páginas de sus "Causas de la guerra de España", librito prologado por Gabriel Jackson, uno de los pocos hispanistas que me merece absoluto crédito en su objetividad. Sugerimos al lector el repaso, uno a uno. Porque verá cómo Azaña cita el problema religioso como una de las fuentes de mayor discordia. La falta de disciplina en las filas republicanas sin lo que no habría victoria posible. La deslealtad del «Eje Bilbao-Barcelona». La hipócrita actitud por parte de la Sociedad de Naciones de ignorar cuanto ocurría en la guerra española. Lo mucho de "racial" que había en "furia con que dos masas enemigas se lanzaron la una contra la otra". Su condena a la propaganda comunista y a sus métodos de captación, con la "protección de los ministerios que ocupaban". La imposibilidad de gobernar y subsistir desde y con los extremistas. Y así un largo rosario de observaciones que siempre tendrán gran valor.

Y es que aquella República de 1936, con los dos polos radicalmente opuestos y con los nombres de Bloque Nacional y Frente Popular, ya no es su República. Como no lo eran los escritos de "El Socialista» animando a llevar a cabo una Revolución como en Rusia. Y argumentando que había que hacer entonces, con la victoria del Frente Popular y Azaña separado del Gobierno, "lo que no se pudo hacer en 1931» (Vd. "EI 
Socialista» de 8 de febrero de 1936). Y perdone el lector que concluya este apartado así: A mí eso de hacer ahora lo que no se pudo hacer antes, me suena a algo.

Si estos y otros valores positivos se dieron durante la República, el lector tiene la plena legitimidad para preguntarse por lo que, por otra parte, constituye el propósito fundamental de estos párrafos. $Y$ es: ¿Entonces, por qué fracasó dicho régimen? A ello vamos.

\section{LOS FACTORES QUE INFLUYERON EN LA CRISIS DE LA REPÚBLICA}

Vaya por delante (para que ningún lector malévolo piense lo contrario) que hablamos de crisis. No de final más o menos repentino. En crisis se puede estar mucho tiempo. Al igual que Unamuno nos recuerda que también en agonía, que significa lucha. $Y$ aclara el egregio Rector de Salamanca: lucha contra la muerte, pero también lucha contra la vida. Elija quien leyere. La situación de la República en 1936, tras la victoria electoral de un Frente Popular, integrado por todas las fuerzas de la izquierda, contra un Frente Nacional, igualmente agrupando a todas las de centro y derecha, origina consecuencias harto peligrosas. En un nuevo bandazo se vuelve a las reformas realizadas durante el primer bienio, pero ahora llevándolas a puntos mucho más extremistas. La retirada de Azaña a la Presidencia de la República, por voluntad absolutamente invariable, siendo el personaje que quizá pudiera haber contenido el frenesí revolucionario que no oculta sus aspiraciones de "hacer en 1936 lo que no se pudo realizar en 1931", deja una huella muy seria en la política española. Con el débil Casares Quiroga al frente del gobierno poco podía esperarse. De aquí que en forma acelerada, el desorden y los abusos se hacen dueños de la calle. La España de la chaqueta y corbata y la España del mono y la alpargata aparecen trágicamente enfrentadas. $Y$ viene la razón de Machado anunciando al españolito que viniera al mundo que una de la dos Españas habría de helarle el corazón. Muy evidentemente las dos Españas, nuestro secular y trágico dilema, están ya en la calle.

Pero si todo esto ocurre en el primer semestre de 1936, los factores de la crisis habían tenido nacimiento bastante antes. ¿Cuáles? A mi entender los que, para evitar el agobio del lector, sintéticamente paso a citar. 


\section{a) Una Constitución no integradora}

No consiguió, desde los mismos momentos de su elaboración, esa gran labor de integración de la sociedad o de gran parte de ella que toda Ley de Leyes ha de llevar a cabo si no quiere quedarse en papel mojado, muerto u olvidado. $Y$ la no integración se produce, fundamentalmente, por dos razones. En primer lugar, se trató de una Constitución desfasada. Cuando en gran parte del constitucionalismo moderno se estaba optando por el Ilamado "reforzamiento del Ejecutivo", el texto de 1931 insiste en la influencia revolucionaria francesa, del gobierno de asamblea. La asamblea todo lo podía y de ella dependía continuamente tanto la formación cuanto el mantenimiento de los gobiernos. Los modernos recursos a través de los cuales dichos gobiernos se fortalecen y pueden llevar a cabo una labor más o menos extensa, estaban ausentes. Piénsese, por ejemplo, en la moción de censura constructiva, por no citar nada más que un ejemplo. Y esta hegemonía de la asamblea alcanzaba incluso al Presidente de la República, que de su seno nacía y cuyas atribuciones eran igualmente cicateramente reguladas, tal como ocurrió en la forma empleada para destituir al Presidente Alcalá Zamora, algo harto discutible jurídicamente. $Y$, en segundo lugar, hablamos de incapacidad de integrar, fundamentalmente por haber incluido en su articulado, principalmente en sus artículos 26 y 27 , una regulación claramente parcial del tema religioso. Lo que debió quedar en declaración de que el Estado no tenía religión oficial y, por ende, se producía la normal separación entre Iglesia y Estado; pasó a ser una rigurosa contemplación con características claramente discriminatorias de las órdenes y congregaciones religiosas que perturbó profundamente la vida del país.

Desde la aprobación de los citados artículos y, sobre todo, desde su posterior desarrollo, el gran sector católico de la nación empezó a considerar que aquella Constitución no era su Constitución, levantándose la bandera de su reforma. Si ésta no se realizó durante los últimos tiempos del segundo bienio, a pesar de la propuesta gubernamental, fue porque los acontecimientos desbordaron el final de dicho bienio. Sin embargo, lo importante es que ni el sentimiento, ni el apego constitucional estuvo vigente para muchos españoles que estimaron perseguidas sus creencias. Con el transcurso de los años, el mismo Azaña, que había cedido en este punto a las presiones de la minoría radical-socialista en el período constituyente, dejó escrito su reconocimiento del error cometido. Pero todo resultaba ya tarde. 


\section{b) Un difícil sistema de partidos}

Desde la perspectiva de nuestros días y, sobre todo, gracias a los trabajos de Sartori o a las puntualizaciones del gran maestro García Pelayo (vid. Manuel García Pelayo: "Consideraciones sobre las cláusulas económicas de la Constitución», en el libro colectivo "Estudio sobre la Constitución Española de 1978», edit. por Manuel Ramírez, Libros Pórtico, Zaragoza, 1979), sabemos bien que la vigencia de una Constitución y la estabilidad de un régimen están estrechamente unidas a una serie de factores conexos. Entre ellos, el sistema de partidos vigente y el grado de socialización política del régimen. Vayamos por orden.

Tres son los rasgos mediante los cuales querríamos dibujar brevemente el sistema de partidos que la II República conoce y sobre los que descansa el juego político entre 1931 y 1936:

1. Debilidad del sistema. Rasgo que acompaña a los partidos políticos tanto al comienzo cuanto a lo largo de los años de vida del régimen republicano. Como en otro lugar desarrollamos hace algún tiempo, el régimen se encontró y vivió con partidos creados con precipitación, a veces con la única intención de ayudar a la empresa de traer la República, de escasa o nula estructura organizativa, lastrados por el personalismo y con no poca falta de coherencia en sus alternativas programáticas. Los entonces llamados "partidos republicanos" o "auténticamente republicanos» fueron buen ejemplo de ello. Estamos ante "el partido de Azaña" o "el de Alcalá Zamora" o ante "la ORGA de Casares Quiroga». Partidos que en parte no supieron desprenderse de su inmediato origen en círculos de intelectuales o que en parte siguieron funcionando como plataformas políticas de "personalidades" republicanas. Incluso en los que fueron grandes partidos faltó organización y estructura de partido moderno. El Partido Socialista Obrero Español fue el único partido seriamente organizado al advenir la República. Luego, con el paso del tiempo, la derecha encontró otra excepción: la CEDA. Pero siempre, la debilidad de numerosos partidos influyó no poco en el conjunto del sistema republicano. En unas ocasiones fue causa de fracasos electorales. En otras, motivó la azarosa vida de uniones y escisiones con la que vivió, del comienzo al fin, la vida republicana de partidos. Y continuamente, tuvo su reflejo en los avatares de las minorías parlamentarias, en la aparición de francotiradores y en la falta de disciplina a todos los niveles. Esto se refleja en el hecho de la existencia de 16 gobiernos, un total de 88 Ministros y una permanente inestabilidad.

2. Pluripartidismo extremo y polarización. Excesivo número de partidos que dificultan el normal desarrollo del juego político y parla- 
mentario. $Y$, entre ellos, con presencia de partidos fuertemente opuestos en función del cleavage religioso. Fue el sentido de la CEDA, como gran partido de orientación clerical, y, en parte, del Partido Radical-Socialista como lo contrario. Y con la presencia (y difícil convivencia) de partidos antisistema que hablaban de "superar la República» por la derecha o por la izquierda. Todo esto ocasionó que, en muchos casos, la oposición no lo fuera sobre temas concretos, sino sobre principios, y que los partidos que creían en "aquella» República hubieran de desplegar una actitud defensiva en doble frente. Excesivo pluripartidismo con distancias ideológicas a veces irreconciliables, con polarización y, para mayor complicación, con altos índices de fragmentación entre los mismos partidos que apoyaban el régimen. Todo ello tenía por fuerza que acabar en permanente inestabilidad por inflación en la dialéctica política de oferta y demanda y por la continua necesidad de coaliciones que nacían con escaso futuro de permanencia.

3. ${ }^{\circ}$ Solapamiento de una doble estructura de partidos, la nacional y la regional. Rasgo motivado, claro está, por la existencia del tema regional, que supuso la plena legitimación de partidos políticos con alternativas y alcance regional. Pero el problema se agudizó por la no correspondencia entre esta doble estructura, ni en el terreno ideológico ni en el grado de polarización. La dificultad de establecer una estructura homologable entre ambos planos, obliga incluso a deslindar dos sistemas coexistentes pero diferenciados de partidos en los que no resulta fácil la simplificación. Está claro que el regionalismo desempeñó el papel de eje diferenciador. Pero se trataba de un eje que, en principio y por sí sólo, no da respuesta definitiva (ni entonces ni después) a la localización en el binomio derecha-izquierda a nivel superior al propiamente regional. El Partido Nacionalista Vasco fue autonomista, por decirlo de algún modo, pero se unía a las derechas en gran parte de las votaciones de las Constituyentes. Cataluña conoció la disputa entre Lliga y Esquerra. Y, por terminar con los ejemplos, la Derecha Regional Valenciana no significaba exactamente los mismo, a nivel de espectro ideológico, que su hermana mayor la CEDA. Todo esto ocasionó no pocas complicaciones tanto en el juego parlamentario como en las políticas electorales. Porque, para que nada resultara fácil, tampoco fue coincidente el tempo del desarrollo político en ambos niveles. El del juego de fuerzas catalán, por ejemplo, no se correspondió con el nacional, como quedó claro en las incidencias del segundo bienio.

Es posible que, al final de la República, se hubiera iniciado un proceso de mayor acercamiento entre partidos que habría facilitado un sistema de fuerzas políticas y un juego político más coherente que quedaron traumáticamente frustrados. Pero lo cierto es que la II República 
vivió toda su singladura histórica lastrada por un juego político cuyos protagonistas antepusieron demasiadas cosas a la permanencia del consenso republicano, por lo demás ya demasiado frágil. Sólo al final, cuando era la misma República la que se puso en peligro, se empezó a ver la luz. Hasta entonces, la inevitable política de alianzas y coaliciones, insoslayable ante tal debilidad, no pudo originar otra cosa en un contexto de posiciones tan contradictorias. Lo que se originó fue una política concreta que acabó por no contentar ni a tirios ni a troyanos, como continuamente se puso de manifiesto en la vida de la segunda República.

\section{c) Un muy escaso grado de socialización política}

Nos referimos en este punto a la ausencia de un auténtico esfuerzo por parte de todas las instancias del régimen en la gran tarea de que los valores republicanos calaran en el conjunto de la sociedad. Quizá porque no se tuvo conciencia de esta importancia. Quizá porque faltó tiempo para ello. Pero lo cierto es que si, para sus protagonistas, lo que llegaba con la República era nada más y nada menos que la democracia, ésta, en su doble vertiente de forma de encauzar las reglas del juego político y de filosofía o forma de pensar, no es algo que cae del cielo.

En nuestros días, y como consecuencia de toda la temática de la democracia que el mundo occidental estudia y difunde tras el final de la segunda guerra mundial, sabemos que nadie nace demócrata, sino que se hace demócrata. Y que hacerse demócrata consiste en asimilar unos valores propios de tal principio legitimador de regímenes políticos en el mundo contemporáneo. Son la aceptación de la alternancia en el poder, el indiscutible valor del sufragio y la posibilidad tanto de elegir gobernantes cuanto de exigirles responsabilidad política por su actuación. Es lo básico. Pero también sabemos que a ello hay que añadir la conformación de una manera de ser y de actuar, que se opone a la autoritaria, y que descansa en la creencia en el diálogo, la aceptación del distinto y de lo distinto, la asimilación de la relatividad de la verdad política, etc. Fuimos pioneros en el estudio de este tema para nuestra actual democracia y a ello nos remitimos (vid. Manuel Ramírez: "La socialización política en España: una empresa para la democracia», Revista Sistema, n. $\left.{ }^{\circ} 34,1980\right)$.

Pues bien, esta tarea, sin la que ningún régimen puede aspirar a durar sin el continuo empleo de la fuerza, tuvo muy escaso reflejo durante los años republicanos. Había llegado la República, sí. Pero qué valo- 
res, qué creencias era preciso inculcar y difundir a través de todas las llamadas agencias de socialización. Desde la familia a la escuela. Desde la prensa al partido político. En esto se reparó muy poco. La gran tarea creativa en lo cultural y en lo científico que la República llevó a cabo y de la que hemos hablado, no incluía esta necesaria labor socializadora en los valores del régimen.

La excepción, como casi siempre, la constituyó Manuel Azaña. El único político que, una y otra vez, puso el dedo en la llaga señalando esta necesidad.

En octubre de 1931 y cuando defendía en las Cortes el proyecto de Ley de Defensa de la República, se formulaba Azaña estas preguntas: "¿Es que podemos consentir que continúe haciéndose este vacío en torno a la institución pública que hemos venido a instaurar, para que cunda en las masas españolas la persuasión de la impotencia del régimen republicano, de la ineficacia de las instituciones republicanas? ¿Es que podemos olvidar que al cabo de siete meses de régimen nos encontramos todavía con que en una inmensa cantidad de pueblos y de aldeas la República no ha penetrado?" (vid. Diario de Sesiones de Cortes de 20 de octubre de 1931).

$Y$ tres años más tarde, insistiendo en la necesidad de extender la penetración de la República, hablaba así a los jóvenes de su partido: "La República no es un texto de derecho político; la República está hecha para los españoles y está hecha en España, y por eso la llamamos República española, con nombre y faz y apellido y acento. Digo, por tanto que esta formación del espíritu republicano, imbuido desde la juventud, conocido y admitido desde la juventud, esta solidaridad, percepción, afición y apego a engrandecer los valores eternos, permanentes y universales con que el nombre de España se ha incorporado a la civilización universal es una pieza principal, capital y fundamental. Sin eso la sociedad española continuaría existiendo, seguro; habría este o el otro régimen político, pero sería semejante al de un arrecife que surge sobre las olas y millones de seres lo sostienen sin saber cuál es su función» (vid. Manuel Azaña: "Discurso a los jóvenes revolucionarios", pronunciado en el Coliseo Pardiñas de Madrid el 16 de abril de 1934 y recogido en Obras Completas, vol. II, México, 1966).

Cuando Azaña habla de República está volviendo a identificar los términos de República y Democracia. Si en ello pensamos, las palabras del jefe de Izquierda Republicana, en el discurso reproducido, constituyen toda una invitación a la tarea de socializar en democracia. Y una democracia fuertemente ligada a su permanente idea de una España moderna. 
Lo que ocurre es que poco más se hizo. Y la socialización en democracia es mucho más, naturalmente. $Y$ en esta tarea, por demás, los intelectuales casi no acompañaron. La decepción les había alejado pronto del ruedo de la política. $Y$, en otros casos, como en el de Unamuno (nada menos que Ciudadano de Honor de la República) lo que encontramos, cuando más falta hacía su voz y su apoyo, es pura ironía, en parte por no entender la identificación antes citada y en parte porque acaso andaban ya germinando las dudas que luego iba a tener. No de otra forma cabe entender estas palabras publicadas en Ahora nada menos que el 3 de julio de 1936: "Cada vez que oigo que hay que republicanizar algo me pongo a temblar, esperando alguna estupidez inmensa. No injusticia, no, sino estupidez. Alguna estupidez auténtica, y esencial, y sustancial, y posterior al 14 de abril. Porque el 14 de abril no lo produjeron semejantes estupideces. Entonces los más de los que votaron la República ni sabían lo que es ella ni sabían lo que iba a ser "esta» República. ¡Que si lo hubieran sabido...! Y en cuanto a lo de republicano, hace ya cinco años que cada vez sé menos lo que quiere decir. Antes sabía que no sabía yo lo que quiere decir eso; pero ahora sé más, y es que tampoco lo saben los que más de ello hablan». Si cambiamos la palabra República por la "democracia» quizá algo de la terrible afirmación unamuniana siga vigente. Democracia no es ni demagogia, ni igualitarismo en lo mediocre.

\section{d) La debilidad del consenso republicano}

Estamos ante lo que, a nuestro juicio, fue tema crucial durante la vida de la II República y factor determinante de su propia inestabilidad. Desde la actual terminología científico-política podemos definirlo como fragilidad del consenso sobre el que descansó el régimen republicano, en parte motivado por la ausencia de una eficaz socialización política en los valores que a dicho régimen sostenían, como acabamos de ver.

La conquista del consenso fue especialmente difícil para la II República. Ante todo, por la ausencia de acuerdo en la clase de República que se deseaba y que era preciso mantener. La divergencia estuvo desde el principio y en no pocas direcciones apareció a la hora de elegir entre forma de Estado unitario o forma de Estado federal que permitiera la existencia de regiones autónomas, divergencia que traspasaba con creces el debate constitucional y que resultó capaz de movilizar a grandes sectores del país a favor de una u otra postura. Se puso de manifiesto en el instante mismo de definir la forma de gobierno a constituir, cuando se discutía el artículo primero de la Constitución. ¿Repú- 
blica democrática, a secas, o República de trabajadores? Tampoco la discusión era puramente semántica. Ya se sabe que el compromiso fue una República democrática de trabajadores "de toda clase». Pero en los debates estuvo presente el tema de la clase de República que había llegado y aquella otra a la que se quería llegar. La divergencia se hizo tempestad en la discusión de los temas religiosos, motivando incluso las dimisiones de dos protagonistas, Alcalá Zamora y Maura, que siempre habían pensado en otro tipo de República distinta a la que decían venía a dañar sus conciencias de católicos, y que hablaban por boca de muchos españoles. Y en fin, la discusión sobre la clase de República fue el objeto de parte de la derecha y de parte de la izquierda. Para unos, se había ido demasiado lejos. Para otros, no servía aquel modelo de República que llamaban burguesa y que decían no representaba sus intereses y aspiraciones. Pronto surgió también la desilusión entre quienes con más ardor habían fletado la empresa de traer la República a España: los intelectuales. Y pronto también, aunque con muy distinta canción, surgieron los grupos para los que superar, significaba sencillamente arrasar lo existente y crear el "Nuevo Estado".

Ahí residió el gran obstáculo para la consolidación de un sólido consenso y para la tarea de una moderna socialización política en los valores que la República representaba. La ilusión de la primera hora se vio pronto enturbiada por las distintas posiciones que comentamos. Durante el primer bienio, los socialistas hubieron de sacrificar parte de sus aspiraciones para lograr la estabilidad de una alianza con los republicanos. Más tarde, la escisión en el seno del mismo partido empezó a hacer la cuestión más difícil. En los últimos meses de la República, la posición de uno de sus sectores hablaba ya muy claramente de otro tipo de República. Entre los republicanos, tampoco faltaron los recelos por la colaboración con los socialistas y ante el peligro de un tipo de República que no era la que habían deseado. Y en la derecha, era suficiente con la utilización del tópico de que "se estaba haciendo política socialista" para dar rienda suelta a los ataques y para encubrir lo que, en gran parte, estaba detrás de su postura: el deseo de que nada cambiara. No hubo nunca consenso porque nunca hubo una imagen generalmente aceptada del tipo, de la clase de República que existía y se deseaba. Y, así las cosas, los resultados no podían ser otros: inestabilidad y discrepancia entre todos y ante todo. Esta es, sin duda, una de las más permanentes lecciones que hoy podemos recibir de aquella experiencia. 


\title{
Resumen
}

El autor analiza la situación de permanentes escisiones en la primera parte del siglo XX, así como las causas que hacían desear la caída de la Monarquía de Alfonso XIII y la llegada de la Segunda República. En la zona de lo positivo de este régimen sitúa la forma pacífica de la llegada del 14 de abril de 1931, la labor cultural de la República y, de manera muy especial, la importancia de la figura de Manuel Azaña.

Al resumir los factores que originan la crisis republicana, el autor cita la no existencia de una Constitución integradora, el excesivo pluripartidismo que origina la permanente inestabilidad política, la ausencia de socialización en los valores republicanos $y$, finalmente, la falta de consenso sobre la clase de República que unos y otros deseaban.

\section{Palabras clave}

República. - Cultura. - Manuel Azaña. - Constitución de 1931. - Pluripartidismo excesivo. - Socialización política. - Falta de consenso.

\section{Summary}

I. INTRODUCTION. II. THE FACTORS THAT INFLUENCED IN THE CRISIS OF THE REPUBLIC. a) A non integrative Constitution. b) A difficult system of parties. c) A very scarce grade of political socialization. d) The weakness of the republican consent.

\begin{abstract}
The author analyzes the situation of permanent divisions in the first part of the XX century, as well as the causes that made want the fall of XIII Alfonso's Monarchy and the arrival of the Second Republic. In the area of the positive thing of this régime it locates the peaceful form of the arrival of the day April 14 1931, the cultural work of the Republic and, in a very special way, the importance of Manuel Azaña's figure.
\end{abstract}

When summarizing the factors that originate the republican crisis, the author it mentions the non existence of an integrative Constitution, the excessive number of political parties that originates the permanent 
political uncertainty, the socialization absence in the republican values and, finally, the consent lack on the class of Republic that some and others wanted.

\section{Key words}

Republic, Culture, Manuel Azaña, Constitution of 1931, excessive number of political parties, political Socialization, consent Lack. 